\title{
Bone vascularization and bone quality (composition) as predictive factors for early marginal bone loss around implants. A retrospective evaluation.
}

IMPLANT THERAPY OUTCOMES, PERI-IMPLANT BIOLOGY ASPECTS
De Winter S., Debaere H., Cox C., Coucke W., Teughels W., Quirynen M.

Department Oral Health Sciences, Periodontology, KU Leuven, Leuven, Belgium

\section{Abstract}

Aim:

The aim of this study was to evaluate if the amount of bleeding of the osteotomy site and/or the bone quality (cortical vs. cancellous) has an impact on early implant failure and bone remodeling

\section{Methods and materials:}

The outcome of 1701 implants was evaluated retrospectively via radiographs at implant placement, abutment connection and 1 year loading. The vascularization of the bone was assessed subjectively at the osteotomy site during implant placement by the surgeon (good, medium and poor vascularized bone). The bone quality was determined by the relative proportion of cortical and trabecular bone at the insertion site, analyzing CBCTs. Other parameters like primary stability, ISQ values, dehiscence, submerged healing, smoking, and position of the implant were also recorded

\section{Results:}

For submerged implants there was significantly more bone loss between mplant placement and abutment placement in less vascularized bone. During the first year of loading an additional impact of vascularisation was not observed.

There was significantly less bone loss at osteotomy sites with more cancellous bone, both between insertion and loading, and during the first year of loading.

\section{Conclusion:}

Within the limitations of this retrospective study one might conclude that the amount of vascularization of the osteotomy site and the proportion of cancellous bone has an impact on the early healing after implant surgery.

\section{Background and Aim}

The success of a prosthetic rehabilitation with implants relies to a great extent on the interface between the implant and the bone. Bone loss around implants may have several causes. As reported in the literature, surgical trauma, implant design, occlusal overload, bone density, establishment of an appropriate biological width, development of a pathogenic biofilm, and failure in the blood supply are the key-factors nvolved (Traini et al. 2006) ${ }^{1}$. The biological role of blood vessels in the homeostasis of the bone goes far beyond the transportation of nutrients, oxygen, numerous cell types, and the removal of waste products to and from all tissues in the body (Mamalis et al. 2011)2.

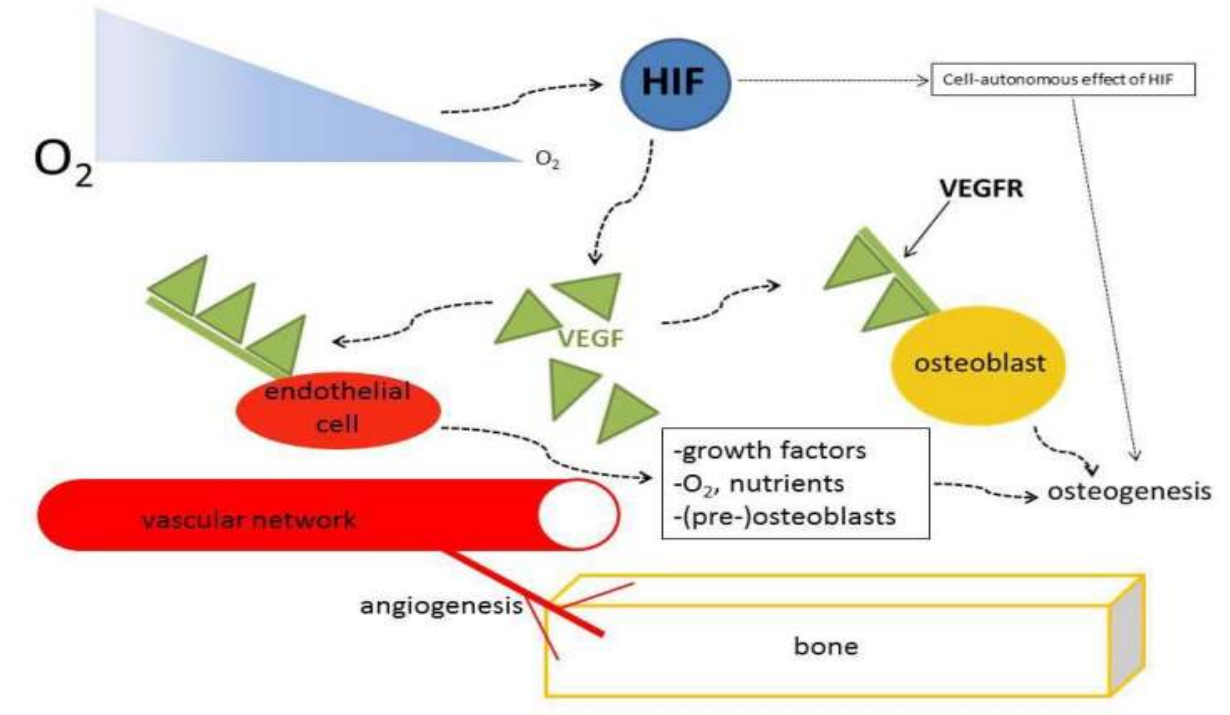

VEGF: vascuair endothelial growth factor HIF: hypoxia-inducabale factors

\section{Methods and Materials}

This retrospective cohort study was performed on 636 consecutive patients, receiving a total of 1701 implants.

Parameters:

Bone vascularization estimation: type $1=$ good,

type $2=$ medium and type $3=$ poorly vascularized bone

\section{Bone quality estimation:}

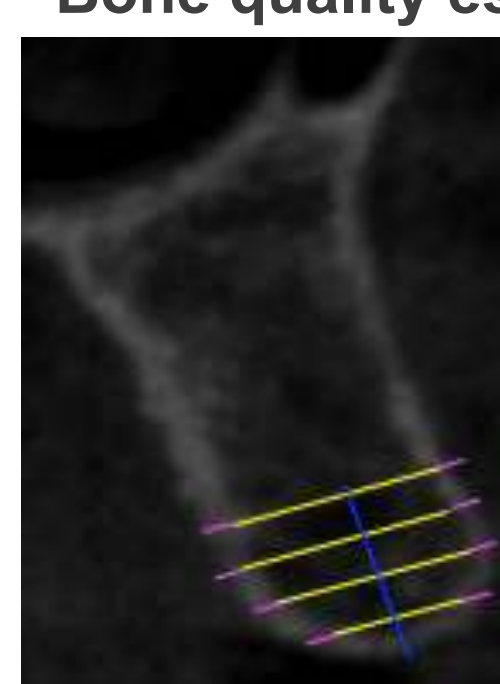

The width of the cortical and cancellous bone were measured, respectively, $1,3,5,7 \mathrm{~mm}$ away from the crest. From the recorded measurements, following properties were calculated: total width of the bone in which the implant was placed, propotion of cotical and cancellous bone in percentage per location bone per implant insertion site.

Bone level changes:

- between implant insertion and abutment connection ( $\Delta$ ins $-A b)$ - between abutment connection and 1 year after implant loading $(\Delta$

- between implant placement and 1 year loading $(\Delta$ ins $-1 y)$

\section{Statistical analysis:}

For each possibly explanatory variable separately, a linear mixed model was set up to assess the relation between the variable and bone loss with patient as random factor and the variable itself as fixed factor. The analysis then continued by interpreting the regression coefficient for continuous variables and comparing the groups with a correction for simultaneous hypothesis testing according to Tukey for the discontinuous variables.

\section{Results}

- Bone vascularization: in the early stages of healing there is significantly more bone loss in the group with medium and poor vascularization. After abutment connection there is almost no noticeable difference between the groups.

\begin{tabular}{c|c|c|c|} 
& $\Delta$ ins $-\mathrm{Ab}$ & $\Delta \mathrm{Ab}-1 \mathrm{y}$ & $\Delta$ ins $-1 \mathrm{y}$ \\
\hline $\begin{array}{c}\text { good } \\
\mathrm{n}=678\end{array}$ & $-0.22( \pm 0.64)$ & $-0.51( \pm 0.75)$ & $-0.75( \pm 0.92)$ \\
& $(-1.48-1.04)$ & $(-1.98-0.97)$ & $(-2.55-1.05)$ \\
& & & \\
medium & $-0.35( \pm 0.53)$ & $-0.45( \pm 0.76)$ & $-0.76( \pm 0.97)$ \\
$n=107$ & $(-1.41-0.70)$ & $(-1.96-1.05)$ & $(-2.68-1.16)$ \\
& & & \\
\hline $\begin{array}{c}\text { poor } \\
n=14\end{array}$ & $-0.83( \pm 0.79)$ & $-0.52( \pm 1.05)$ & $-0.76( \pm 0.88)$ \\
& $(-2.76-1.1)$ & $(-3.07-2.04)$ & $(-2.66-1.13)$ \\
& & &
\end{tabular}

- Bone quality: Bone loss after 1 year of loading was significantly less in more spongious bone $(p=0,0014)$.

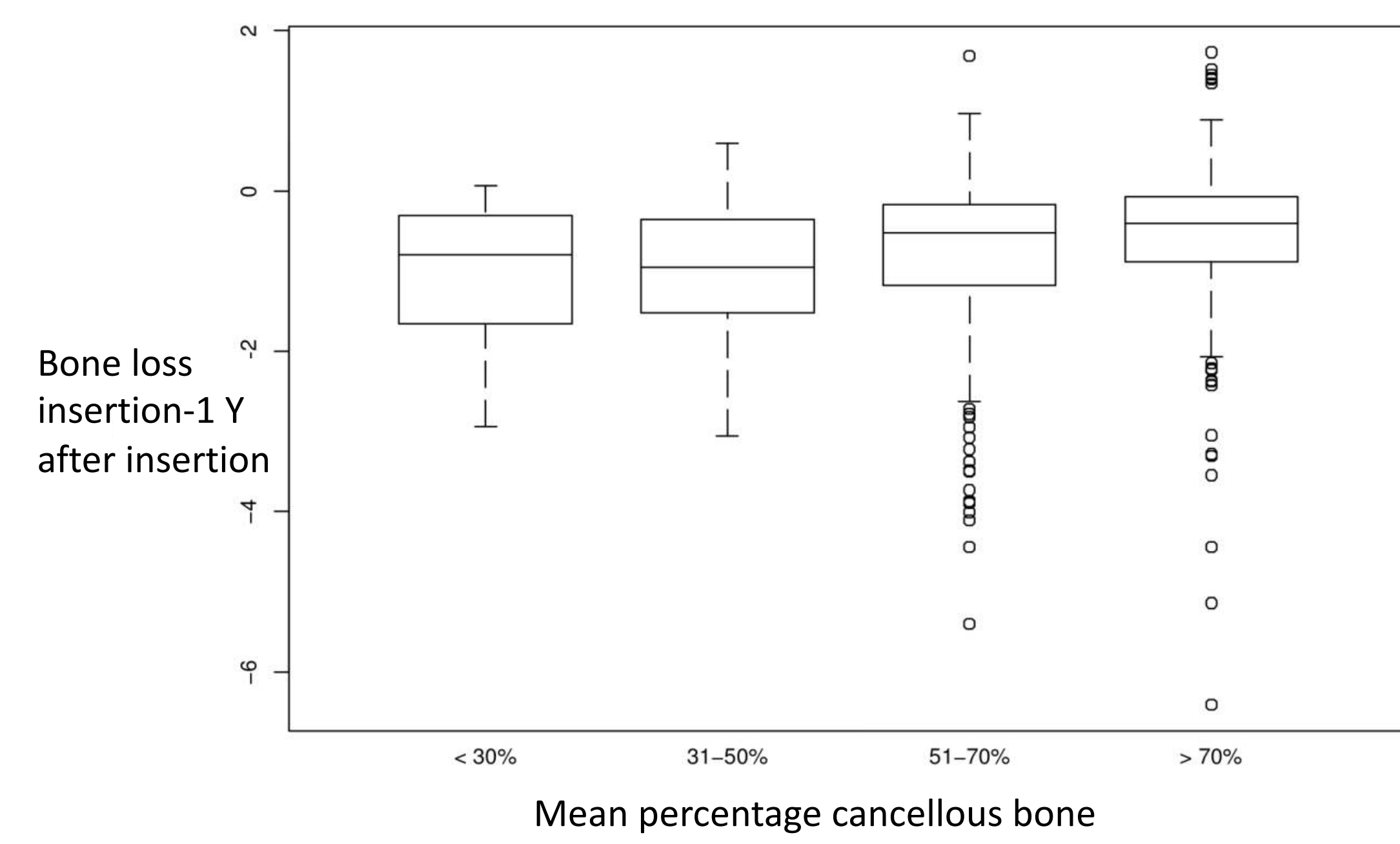

Mean percentage cancellous bone

\section{Conclusions}

Davies (2003) 3 stated that trabecular bone, previously characterized as "poor quality" bone is far better adapted to rapid healing than cortical bone. In the present study, more bone loss was observed in more cortical bone.

Within the limitations of this retrospective study one might conclude that the amount of vascularization of the bone and the proportion of cortical vs. spongious bone has an impact on early peri-implant bone loss. In the planning of oral implants one should analyze the cortical vs. cancellous ratio and if possible try to place the implants in cancellous, more vascularized bone, to maintain the marginal bone level.

\section{References}

Traini T., Assenza B, San Roman F. Thams U Caputi S., and Piattelli A., "Bone microvascular pattern around loaded dental implants in a canine model.," Clinical oral investigations, vol. 10, no. 2, pp. 151-6, Jun. 2006.

2. Mamalis A.A. and Cochran D.L., "The therapeutic potential of oxygen tension manipulation via hypoxia inducible factors and mimicking agents in guided bone regeneration. A review.," Archives of oral biology, vol. 56, no. 12, pp. 1466-75, Dec. 2011.

3. Davies J.E. "Understanding peri-implant endosseous healing." J Dent Educ. 2003 Aug;67(8):932-49 